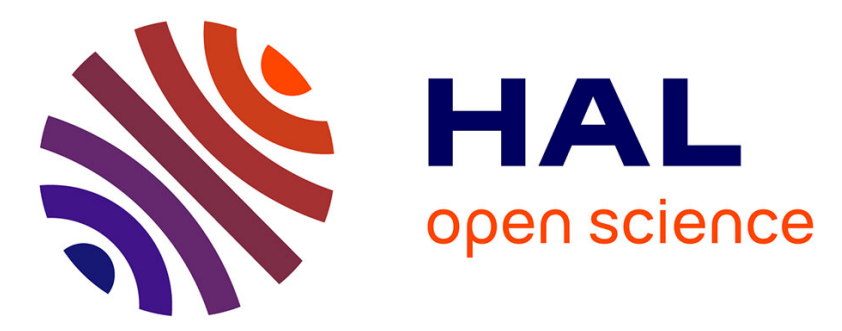

\title{
Polycyclic aromatic hydrocarbon transformation with laccases of a white-rot fungus isolated from a Mediterranean schlerophyllous litter
}

Anne Marie Farnet da Silva, G. Gil, F. Ruaudel, A. C. Chevremont, E. Ferre

\section{- To cite this version:}

Anne Marie Farnet da Silva, G. Gil, F. Ruaudel, A. C. Chevremont, E. Ferre. Polycyclic aromatic hydrocarbon transformation with laccases of a white-rot fungus isolated from a Mediterranean schlerophyllous litter. Geoderma, 2009, 149 (3-4), pp.267-271. 10.1016/j.geoderma.2008.12.011 . hal02071817

\section{HAL Id: hal-02071817 https://hal.science/hal-02071817}

Submitted on 18 Mar 2019

HAL is a multi-disciplinary open access archive for the deposit and dissemination of scientific research documents, whether they are published or not. The documents may come from teaching and research institutions in France or abroad, or from public or private research centers.
L'archive ouverte pluridisciplinaire HAL, est destinée au dépôt et à la diffusion de documents scientifiques de niveau recherche, publiés ou non, émanant des établissements d'enseignement et de recherche français ou étrangers, des laboratoires publics ou privés. 


\title{
Polycyclic aromatic hydrocarbon transformation with laccases of a white-rot fungus isolated from a Mediterranean schlerophyllous litter
}

\author{
A.M. Farnet ${ }^{\mathrm{a}, *}$, G. Gil $^{\text {b }}$, F. Ruaudel ${ }^{\text {a }}$, A.C. Chevremont ${ }^{\text {a }}$, E. Ferre ${ }^{\text {a }}$

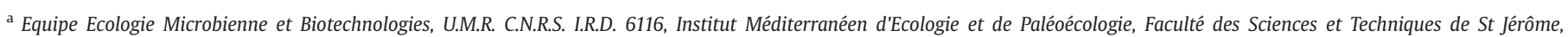 \\ Université Paul Cézanne, Marseille, France \\ ${ }^{\mathrm{b}}$ Equipe Biocatalyse, U.M.R. C.N.R.S. 6180, Faculté des Sciences et Techniques de St Jérôme, Université Paul Cézanne, Marseille, France
}

Keywords:

Abiotic oxidations

Bioremediation

Ionisation potential

Mediator

Phenoloxidases

Polycyclic aromatic compounds

\begin{abstract}
A B S T R A C T
This study was conducted to investigate whether a laccase from a white-rot fungus isolated from Mediterranean forest litters was able to oxidise widespread pollutants such as polycyclic aromatic hydrocarbons (PAH). Six different isoforms from the constitutive laccase of Marasmius quercophilus strain19 were revealed using isoelectric focusing. In vitro studies showed that these laccase isoforms were able to transform anthracene and benzo(a)pyrene while naphthalene and phenantrene were not oxidised. This result on PAH transformation is linked to the ionisation potential (IP) of PAH: the laccases under study are able to oxidise PAH with IP $<7.55 \mathrm{eV} .2,2$ '-azino-bis-3-ehtylbenzothiazoline-6-sulfonicacid (ABTS) and hydroxybenzotriazole (HBT), were also used as mediators: percentages of anthracene and benzo(a)pyrene transformation were higher when ABTS was used and when mediators were pre-oxidised with laccases whatever the mediator used. Moreover, after an eight or fifteen-day culture of strain 19 with anthracene, the percentage of disappearance was 73 and $79 \%$ respectively. This study also highlights the fact that ABTS or one of its oxidised form, ABTS radical cation $\left(\mathrm{ABTS}^{+}\right)$, can be respectively oxidised or reduced through abiotic reactions. Thus, ABTS, because of its electrochemical characteristics, can hardly be considered as a relevant substrate to measure laccase activities in complex environments such as soils or litters where various abiotic reactions are likely to occur.
\end{abstract}

\section{Introduction}

Mediterranean forests are mainly composed of schlerophyllous species such as Quercus ilex L. because of climate conditions characterised by draught in summer. Thus particularly high amount of various phenolic compounds can be found in forest litters in this area (Racon et al., 1988). Under these drastic conditions, certain microbial species -mainly white-rot fungi- are able to transform phenols, which are recalcitrant molecules, via phenoloxidases such as laccases. For instance, Marasmius quercophilus is a white-rot basidiomycete which extensively colonises evergreen-oak litter and produces several laccases (Farnet et al., 2000, 2004). These blue copper oxidases are indeed polymorphic: different isoforms of laccases can be produced depending on environmental conditions and on fungal species (Palmieri et al., 2000, 2003; Baldrian, 2003). Thus Mediterranean forest litters may be considered as a source of laccase diversity because of their specific chemical composition.

Furthermore, in the Mediterranean area, anthropogenic pressure is particularly high and do not favour the ecosystem functioning. Various organic pollutants can greatly affect organic matter turn-over in soils or

\footnotetext{
* Corresponding author. Tel./fax: +33 491288190.

E-mail address: a-m.farnet@univ-cezanne.fr (A.M. Farnet).
}

litters: for instance, polycyclic aromatic hydrocarbons can drastically alter microbial activities (Hamdi et al., 2006; Eom et al., 2007). These widespread pollutants are recalcitrant to biotransformation because of their aromatic and condensed structure leading to a very low solubility (Muhit et al., 2008). Laccases produced by white-rot fungi have been widely used in various detoxification processes since they oxidise aromatic pollutants such as chlorophenols, PAH or dyes (Bezalel et al., 1996; Potin et al., 2004; Zhang et al., 2008). Moreover, the effects of mediators on oxidation potential of laccase have also been extensively investigated: these molecules are commonly used in laccase reaction since they can enlarge laccase substrate range. Mediators are oxidised by laccases and then oxidise other compounds in a non-specific reaction. Although synthetic mediators, such as 2,2'-azino-bis-3-ehtylbenzothiazoline-6-sulfonicacid (ABTS) and hydroxybenzotriazole (HBT), are usually used as models, other studies have focused on natural phenolic compounds as mediators since it can provide information about their potential role in natural environments (Calcaterra et al., 2008).

In this work we wanted to investigate the oxidation potential of laccase functional diversity towards PAH. Tools which can rapidly discriminate the various laccase isoforms are still lacking. Based on a previous work (Farnet et al., 2000), isoelectric focusing (IEF) appeared to be a promising method. Here, we used this technique to investigate laccase diversity of a $M$. quercophilus strain and to characterise these 
isoforms reactivity towards different substrates such as ABTS which can also be used as a mediator. The potential of PAH oxidation of these isoforms has indeed been investigated with or without mediators (ABTS or HBT) in order to assess the role of such diversity in natural ecosystem contaminated with these pollutants. Moreover this study has also focused on the importance of mediator pre-oxidation to test whether preoxidation by laccases favour PAH oxidation yields.

\section{Materials and methods}

\subsection{Isolation of the strain}

Strain 19 of M. quercophilus was isolated from a part of the site of La Gardiole de Rians, Var, France (1 ha) using the rhizomorphic form of the fungus (Farnet et al., 1999). A fungal cap culture was firstly grown on a malt extract agar medium (MEA), (malt extract, (Bio Mérieux, France): $20 \mathrm{~g} \mathrm{~L}^{-1}$, agar (Bio Mérieux, France): $20 \mathrm{~g} \mathrm{~L}^{-1}$ ) supplied post-autoclaving with chloramphenicol, (Sigma, France): $50 \mathrm{mg} \mathrm{L}^{-1}$. Secondly, the pure mycelial culture obtained was used to inoculate an agar medium containing whole wheat flour (Bio Mérieux, France: $20 \mathrm{~g} \mathrm{~L}^{-1}$ ) which favours rhizomorph production. Then one rhizomorph was used to inoculate a MEA plate to obtain a pure dicaryotic culture.

\subsection{Culture conditions and partial purification of laccase}

Precultures were performed in $200 \mathrm{~mL}$ Erlenmeyer flasks with $50 \mathrm{~mL}$ of malt extract liquid medium (MEL) containing malt extract $\left(20 \mathrm{~g} \mathrm{~L}^{-1}\right)$ and $\mathrm{CuSO}_{4}\left(5 \mathrm{~g} \mathrm{~L}^{-1}\right)$. They were inoculated with a plug $(1 \mathrm{~cm}$ diameter) from a fungal culture grown on MEA and incubated at $25{ }^{\circ} \mathrm{C}$ in darkness for 4 days. These precultures were used to inoculate two 3 L-Erlenmeyerflasks with $400 \mathrm{~mL}$ of MEL. Cultures were incubated at $25^{\circ} \mathrm{C}$ for 5 days in darkness under shaking at $200 \mathrm{rpm}$.

Enzyme activity was measured by following the oxidation of syringaldazine [N,N'-bis-(3,5-dimethoxy-4-hydroxybenzylidene)hydrazine] to quinone $\left(\varepsilon^{\mathrm{M}}=65000 \mathrm{M}^{-1} \mathrm{~cm}^{-1}\right)$ at $525 \mathrm{~nm}$ in acetate buffer $0.1 \mathrm{M}, \mathrm{pH} 4.0$ on a spectrophotometer Kontron Uvikon 860. One unit (U) of laccase activity is defined as the amount of enzyme that oxidises $1 \mu \mathrm{mol}$ of the substrate/min. Sampling (two replicates) was performed from the third to the seventh day of culture. The reaction mixture contained $500 \mu \mathrm{L}$ of the medium, $10 \mu \mathrm{L}$ of syringaldazine $(0.6 \%$ in methanol) and $2.5 \mathrm{~mL}$ of acetate buffer (two replicates for each sampling). Cultures were filtered on a glass microfibre filter GF/D, $2.7 \mu \mathrm{m}$ (Whatman, England). The filtered medium was concentrated using dialysis tubes (Cellu Sep, VWR, France) rated at $10 \mathrm{MW}(10 \mathrm{kDa}$ ) cut-off. A total volume of $100 \mathrm{~mL}$ was obtained. The concentrated filtered medium was loaded on an ion-exchange Mono $\mathrm{Q}$ in fast performance liquid chromatography (FPLC) equilibrated with phosphate buffer $0.1 \mathrm{M} \mathrm{pH}$ 6.0. Laccase was eluted with a step gradient $(0.2 \mathrm{M}, 0.4 \mathrm{M}, 2 \mathrm{M} \mathrm{NaCl})$ for 30 min at a flow

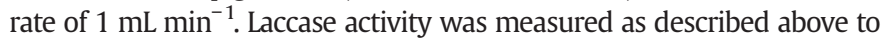
detect fractions containing laccase. Laccase purity was checked on a PAGE (polyacrylamide gel electrophoresis) carried out according to Laemmli (1970) using 4\% stacking gel and 7.5\% separating gel at $220 \mathrm{~V}$ with the Mini-Protean II electrophoresis cell (Biorad) and protein was stained using the Coomassie Blue standard method.

\subsection{Isoelectric focusing of the purified laccase}

Isoelectric focusing was performed using the Biorad Rotofor preparative IEF Cell. The sample was prepared as follow: $10 \mathrm{~mL}$ of the purified enzyme solution were added to $1 \mathrm{~mL}$ of Bio-Lyte 3/10 ampholytes (Biorad) and distilled water q.s.p. $50 \mathrm{~mL}$ to obtain a $\mathrm{pH}$ gradient from 3 to 10. Isoelectric focusing was performed for $3 \mathrm{~h}$ at $200 \mathrm{~V}$ till the $\mathrm{pH}$ gradient gets stable (voltage about $360 \mathrm{~V}$ ). $2 \mathrm{~mL}$-fractions were collected under vacuum and the $\mathrm{pH}$ was immediately measured for each fraction. Laccase activity was also measured for each fraction using syringaldazine as a substrate as described above.

\subsection{Determination of $V_{m}$ and $K_{m}$ for each laccase isoelectric isoforms}

ABTS and 2,6 dimethoxyphenol (DMP) were used as substrates to determine the $V_{\mathrm{m}}$ and $K_{\mathrm{m}}$ which were calculated using the LineweaverBurk transformation of Michaelis-Menten equation. The concentrations ranged from 0.5 to $50 \mu \mathrm{M}$ and from 0.2 to $10 \mu \mathrm{M}$ for ABTS and DMP respectively in acetate buffer $0.1 \mathrm{M} \mathrm{pH} 4.5$.

\subsection{High Performance Liquid Chromatograph (HPLC) analysis of PAH transformation}

HPLC system was equipped with a C18 Reverse Phase column (Merck, $4.6 \times 250 \mathrm{~mm}$ ) in the following gradient system: solvent A, water/trifluoro acetic acid $0.1 \% /$ acetonitrile $90 / 10 \mathrm{v} / \mathrm{v}$, solvent $B$ water/trifluoro acetic acid $0.1 \%$ /acetonitrile $95 / 5 \mathrm{v} / \mathrm{v}$, gradient $=5$ to $15 \mathrm{~min}$, A $100 \%$ to B $100 \%$; 15 to 25 min B $100 \%$ (flow rate $1 \mathrm{~mL} \mathrm{~min}^{-1}$ ). The PAH transformation was realised using the mixture of the six isoforms of laccase since a weak activity was obtained for some isoforms. The reaction mixture $(5 \mathrm{~mL})$ contained ABTS or HBT $1 \mathrm{mM}$, PAH $250 \mathrm{mg} \mathrm{L}^{-1}$ and $5 \mathrm{U}$ of laccase in acetate buffer $0.1 \mathrm{M} \mathrm{pH} 4.5$ and $10 \%$ acetone. The reaction mixtures were incubated at $30{ }^{\circ} \mathrm{C}$ for $6 \mathrm{~h}$ in the dark. A 4-hour preliminary ABTSoxidation was also performed using the same conditions and the $\mathrm{PAH}$ solution in acetone was added after the pre-oxidation of the mediator. An extraction with $5 \mathrm{~mL}$ of diethyl ether was performed for each reaction mixture prior to injection. The organic phase was evaporated and the products of extraction were dissolved in $1 \mathrm{~mL}$ of acetonitrile $(30 \mu \mathrm{L}$ injected). The PAH used were: naphtalene, anthracene, phenanthrene and benzo(a)pyrene. All the PAH were purchased from Sigma. The same reactions were performed without mediators. Controls were also realised using only ABTS or HBT with laccase and using ABTS or HBT with the PAH but without laccase. To test ABTS oxidation via benzo(a)pyrene oxidation products, a control with benzo(a)pyrene and ABTS in a sealed flask saturated with nitrogen was also performed. All these controls were incubated at $30^{\circ} \mathrm{C}$ for $6 \mathrm{~h}$. $P$-hydroxybenzoic acid was used as reference in each injection. 9,10-anthraquinone was also injected in order to confirm the identification of the single oxidative product obtained after anthracene oxidation. Peaks were identified based on matching retention time and UV spectra at $254 \mathrm{~nm}$ with those of anthracene and anthraquinone standards.

\subsection{Cultures of M. quercophilus with anthracene}

Cultures, previously inoculated with $10 \mathrm{~mL}$ of preculture of strain 19 , were performed for 8 or 15 days of culture on a rotary shaker (200 rpm) at $25{ }^{\circ} \mathrm{C}$ in $1 \mathrm{~L}$-Erlenmeyer flasks with $200 \mathrm{~mL}$ of MEL including $50 \mathrm{mg}$ of anthracene. Laccase activities were measured everyday from day 4 with syringaldazine as the substrate as described above. Different control cultures without mycelium were performed: control cultures after autoclaving to assess anthracene evaporation during sterilisation and control cultures incubated in the same conditions and for both times of incubation. Each experiment was performed in three replicates. Cultures were filtered and the mycelium as well as the supernatant was extracted three times with the $200 \mathrm{~mL}$ of dichloromethane. The solvent was then evaporated and the solid extract solubilised in $1 \mathrm{~mL}$ of acetone. $30 \mu \mathrm{L}$ of dilution $10^{-3}$ were injected in the HPLC system. A calibration curve was also performed for anthracene concentrations ranging from $10 \mu \mathrm{M}$ to $60 \mu \mathrm{M}$. Results were expressed as percentages of anthracene disappearance.

\subsection{Statistical analysis}

The non-parametric $U$-test of Mann-Whitney was used to separate significantly different means $(P<0.05)$ of remaining anthracene under the different experimental conditions. These analyses were performed using SPSS 11.0 statistical software (SPSS Inc., Chicago, IL). 


\section{Results and discussion}

After laccase purification from $M$. quercophilus cultures on MEL one band on SDS-PAGE (data not shown) was obtained with a molecular weight of $60 \mathrm{kDa}$ as described previously (Farnet et al., 2008). Isoelectric focusing from the partially purified laccase obtained here shows six isoforms (called isoform 1 to 6 ) with the following $\mathrm{pH}_{i}$ : $2.90,3.05,3.25,3.55,4$ and 4.8 . The diversity of the laccase isoforms highlighted here is consistent with previous studies (Palmieri et al., 2003; Stajic et al., 2006). A previous study on this species of basidiomycete (Farnet et al., 2000) revealed three isoforms with different $\mathrm{pH}_{i}(4,4.2$ and 4.4) from the constitutive isoform and four others $(4.75,4.85,4.95$ and 5.1$)$ from an isoform induced with $p$ hydroxybenzoic acid. This M. quercophilus strain (strain 17) was also isolated from an evergreen oak litter in La Gardiole de Rians (Var, France). However, the present result confirmed that isoelectric focusing can thus be considered as a simple method to describe more precisely enzymatic isoform variations at an intraspecific level. Furthermore, this methodology gives information about the amino acid composition of the protein studied: variations in $\mathrm{pH}_{i}$ indicate that the amino acids of the isoforms and thus the protein sequences are different. These differences may influence the biochemical enzyme characteristics, which we partially described by calculating the $V_{\mathrm{m}}$ and $K_{\mathrm{m}}$ values of these different isoforms using ABTS and 2,6DMP as substrates (Table 1). For all the isoforms (except isoform 5), the $K_{\mathrm{m}}$ measured with 2,6 DMP was higher than that with ABTS and $V_{\mathrm{m}}$ was always higher with ABTS as the substrate. Thus the affinity of isoforms $1,2,3,4$ and 6 for ABTS was stronger than that for 2,6 DMP. Although certain biochemical characteristics, such as molecular weight or optimal $\mathrm{pH}$ of activity, are almost the same for many fungal laccases, laccase affinity for susbtrates is variable. For instance a weak laccase affinity for ABTS has been observed in previous studies: Pozdnyakova et al. (2006) found a higher affinity for 2,6 DMP than for ABTS with a laccase produced by Pleurotus ostreatus. On the other hand, Michniewics et al. (2006) have found that laccase isoforms from Cerrena unicolor have a higher affinity for ABTS. In further studies, a greater amount of each laccase isoform should be obtained in order to describe more precisely the respective role of each enzyme in xenobiotic oxidation. For instance, their different affinities for ABTS may favour or not PAH transformation yield and thus their respective contribution to global laccase activity may have an effect on $\mathrm{PAH}$ oxidation efficiency.

Because most of aromatic pollutants can be dissolved only in organic solvents, the effects of such solvents on laccase activity were assessed in a previous study (Farnet et al., 2008). The authors have shown that the laccases produced by $M$. quercophilus were still active in $10 \%$ acetone. Therefore, these conditions were used in the present study. Here, only anthracene and benzo(a)pyrene were oxidised by laccase (Table 2) with or without mediator (ABTS or HBT). This result may be explained by the fact that in naphthalene and phenanthrene, electronic delocalisation is observable in two or three condensed cycles respectively while anthracene or benzo(a)pyrene cycles do not exhibit this characteristic. This structural characteristic may make

Table 1

$V_{\mathrm{m}}$ and $K_{\mathrm{m}}$ (using ABTS and 2,6-DMP) and specific activity of the different laccase isoforms of the constitutive laccase of Marasmius quercophilus found after isoelectric focusing

\begin{tabular}{llllllll}
\hline & & $\mathrm{pH}_{i}$ & & & & & \\
\cline { 3 - 7 } & & 2.99 & 3.06 & 3.24 & 3.55 & 4 & 4.78 \\
\hline ABTS & $K_{\mathrm{m}}$ & 0.78 & 0.667 & 0.755 & 0.521 & 0.378 & 0.136 \\
\multirow{2}{*}{,6 DMP } & $V_{\mathrm{m}}$ & 7.61 & 7.593 & 8.025 & 6.94 & 4.82 & 3.017 \\
& $K_{\mathrm{m}}$ & 0.911 & 0.988 & 1.13 & 0.813 & 0.12 & 0.285 \\
Specific activity & $V_{\mathrm{m}}$ & 4.766 & 4.372 & 3.462 & 2 & 0.81 & 1.037 \\
& & 1.504 & 0.623 & 0.296 & 0.161 & 0.057 & 0.141 \\
\hline
\end{tabular}

Table 2

Ionisation potential and solubility in water of different PAH and oxidation of these compounds with Marasmius quercophilus laccase

\begin{tabular}{|c|c|c|c|}
\hline $\mathrm{PAH}$ & $\frac{\text { lonisation potential }}{(\mathrm{eV})}$ & $\frac{\text { Solubility in water }^{\mathrm{c}}}{\left(\mathrm{mg} \mathrm{L}^{-1}\right)}$ & $\begin{array}{l}\text { Oxidation } \\
\text { with } M \text {. } \\
\text { quercophilus } \\
\text { laccase }\end{array}$ \\
\hline Naphtalene & $8.1^{\mathrm{a}}$ & 0.3 & - \\
\hline Phenanthrene & $8.19^{\mathrm{b}}$ & 1 & - \\
\hline Chrysene & $7.8^{\mathrm{b}}$ & 0.0018 & ND \\
\hline Anthracene & $7.43^{\mathrm{b}}$ & 0.045 & + \\
\hline Benzo(a)pyrene & $7.23^{\mathrm{b}}$ & 0.0016 & + \\
\hline
\end{tabular}

ND: Non-determined.

a Bernal-Martinez et al. (2007).

b Roseen et al. (1995).

Hammel et al. (1986).

impossible the use of laccases for phenanthrene and naphtalene oxidation. This electronic delocalisation is indeed directly linked to the ionisation potential of a molecule. The ionisation potential of naphtalene and phenanthrene are higher than that of anthracene: respectively 8.1, 8.19 and $7.43 \mathrm{eV}$. Hammel et al. (1986) have shown that lignine peroxidase from Phanerochaete chrysosporium was able to oxidise only PAH with an ionisation potential $<\sim 7.55 \mathrm{eV}$ such as pyrene $(\mathrm{IP}=7.5 \mathrm{eV})$. Field et al. (1996) have found the same results with lignine- and manganese-peroxidases from Bjerkandera sp. Thus, when ionisation potentials are available, they can be used to forecast the ability of a laccase to oxidise a molecule. For instance, M. quercophilus laccase is probably unable to oxidise chrysene (IP $=7.8 \mathrm{eV})$. In further studies, it would be valuable to determine the IP value above which oxidation by the M. quercophilus laccase will be impossible, by testing laccase oxidation of several molecules with IP ranging from 7.5 to $8 \mathrm{eV}$.

The anthracene oxidative product obtained with $M$. quercophilus was 9,10-anthraquinone as revealed by the HPLC elution profile (Fig. 1). No traces of other oxidation products were detected with HPLC. 9,10-anthraquinone was also injected in order to confirm the identification of the single product obtained and both the UV scan and the elution profile corroborate our results. No benzo(a)pyrene oxidation products were detected, although this molecule was actually transformed as revealed by the strong decrease in benzo(a)pyrene amount when laccase was used with ABTS (66\%, average of three experiments, standard deviation under $2 \%$ ). Most studies on PAH transformation with laccase have not clearly identified oxidation products: authors have mainly depicted PAH concentration decrease (Cho et al., 2002; Dodor et al., 2004; Potin et al., 2004; Rodríguez et al., 2004, Valentín et al., 2006). Lau et al. (2003), using spent mushroom compost, have found that PAH transformation led to an increase in phtalate concentration. However, this result was not specifically correlated to PAH transformation since phtalates were also extracted from the compost and the authors suggested that it was, in this case, associated with lignocellulose transformation. Hu et al. (2007) have shown the production of quinones (1,6-benzo(a)pyrene quinone, 3,6benzo(a)pyrene quinone and 6,12-benzo(a)pyrene quinone) as well as different non-identified products. Quinone production for both anthracene and benzo(a)pyrene has been linked to nucleophilic attacks and further non-enzymatic arrangements of the molecule, leading to quinones (Collins et al., 1996).

In our reaction mixtures where ABTS was oxidised by laccase, absorption values increase mainly at $425 \mathrm{~nm}$ and to a lesser extent from 600 to $800 \mathrm{~nm}$ (Fig. 2). When anthracene was added to the reaction mixture, the absorption value observed at $425 \mathrm{~nm}$ strongly decreased as well as those from 600 to $800 \mathrm{~nm}$. The reaction mixture, which was green because of the ABTS radical cation formed (Bourbonnais et al., 1998), turned to an almost transparent mixture. This result shows that anthracene oxidation via ABTS actually occurred simultaneously with ABTS reduction. Furthermore, the 

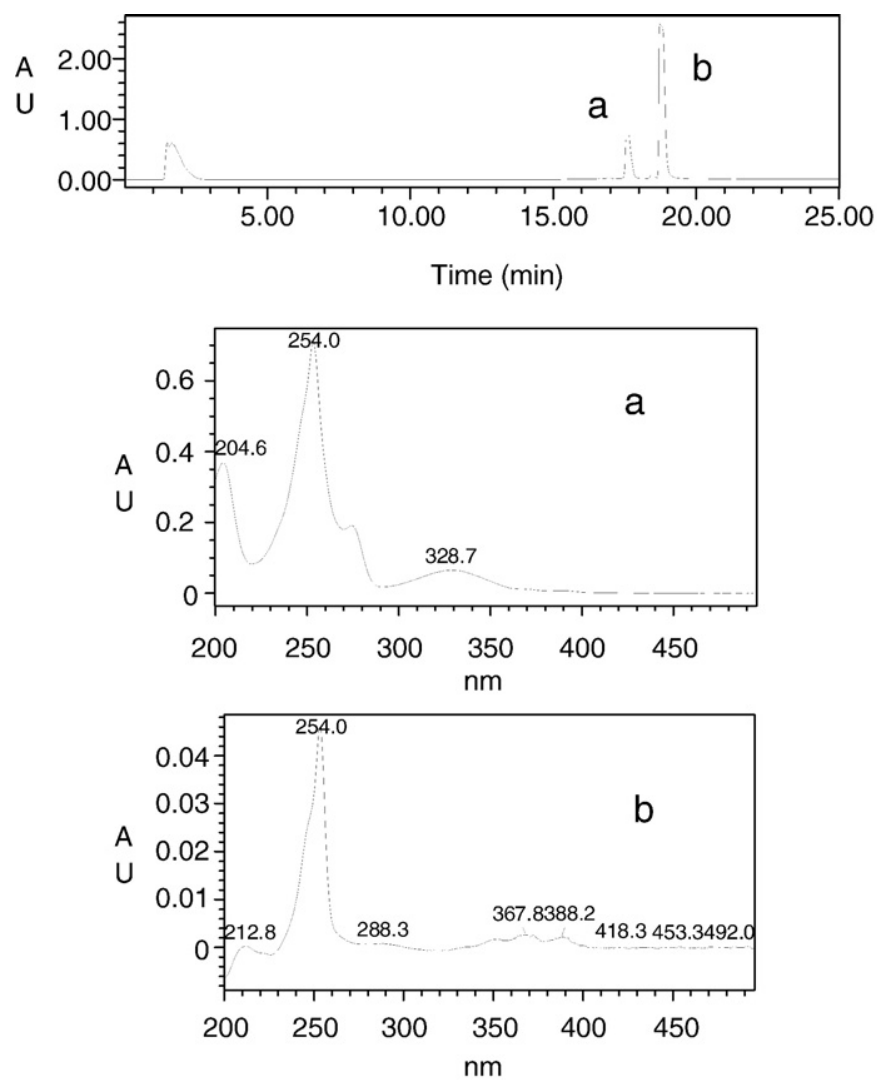

Fig. 1. Elution profile at $254 \mathrm{~nm}$ of the reaction mixture with anthracene and the constitutive laccase of Marasmius quercophilus after extraction and solubilisation in acetone with UV scan of 9,10-anthraquinone and anthracene respectively ( $a$ and b).

remaining anthracene percentages were significantly weakest when ABTS or HBT were previously oxidised as shown in Fig. 3 (respectively 20 and 45\%). Laccases can use both mediators and anthracene as substrates although mediators are considered to be better substrates than anthracene. Thus pre-oxidation of mediators may limit competition between the two molecules and favour anthracene abiotic oxidation with the oxidised form of the mediator (in the case of ABTS, a radical cation as previously described). With benzo(a)pyrene, ABTS reacted differently: the radical cation did not disappear when benzo(a)pyrene was added to the medium. Furthermore, in the

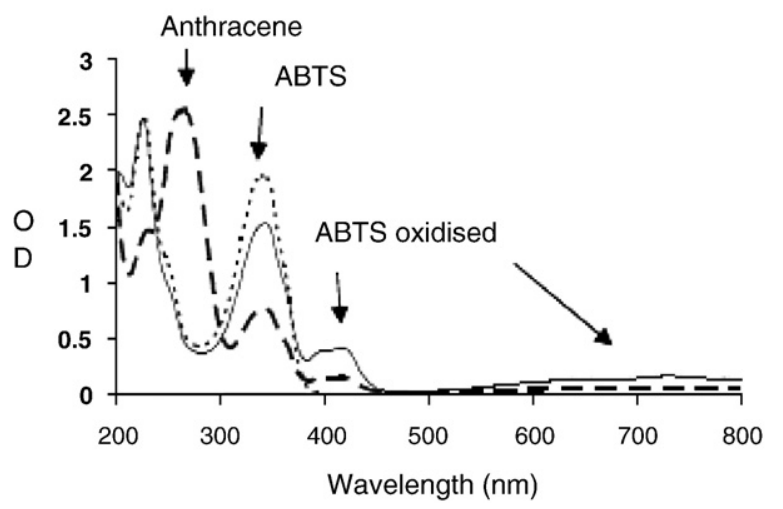

Fig. 2. Spectral scan of the reaction mixture during anthracene oxidation with the constitutive laccase of Marasmius quercophilus: ABTS alone (- - ), ABTS and laccase (-), ABTS, laccase and anthracene (- - ). The arrows indicate the peaks corresponding to ABTS, to the oxidise form of ABTS and to anthracene. reaction control with both ABTS and benzo(a)pyrene, the ABTS radical cation was produced even though no enzyme was added to the reaction mixture. This is probably due to the products of benzo(a)pyrene oxidation with $\mathrm{O}_{2}$ : this PAH has a particularly low IP leading to abiotic oxidation such as photooxidation or oxidation via $\mathrm{O}_{2}$ as previously described (Mill et al., 1981; Miller and Olejnik, 2000). To check this hypothesis, the same experiment (benzo(a)pyrene plus ABTS) was performed in an atmosphere saturated with nitrogen. In this case no ABTS oxidation was observable which is most probably due to the absence of benzo(a)pyrene oxidation by oxygen.

These investigations using ABTS as a mediator highlight the fact that this molecule can hardly be considered as a relevant substrate for laccases under particular experiments. For instance, when laccase activities are detected in soils, many abiotic parameters are likely to cause abiotic oxidation of ABTS or reduction of ABTS radical cations since these molecules are very reactive. In soils contaminated with $\mathrm{PAH}$, abiotic reactions due to $\mathrm{PAH}$ may occur, leading to a misinterpretation of in situ results. Thus in environmental studies, and moreover in contaminated soils, these parameters must be taken into account when measuring laccase activities. ABTS can hardly be a relevant choice as a substrate for laccases because the electrochemical characteristics make ABTS very reactive towards many molecules.

HBT is not an efficient mediator when used with the M. quercophilus laccase under study. This is supported by the fact that HBT redox potential, $E^{0}$, is higher than that of ABTS (1080 and $475 \mathrm{~V} / \mathrm{NHE}$ respectively as described by Baiocco et al., 2003) and oxidation with laccase is always favoured when substrates have low $E^{0}$ since electrons are transferred from the substrate to the laccase active site. Pickard et al. (1999) have also found that HBT was a less efficient mediator than ABTS in anthracene oxidation.

In order to quantify anthracene disappearance in $M$. quercophilus culture, both culture medium and mycelium were extracted (the mycelium was extracted to check whether any adsorption occurred). Furthermore, we took into account anthracene loss after sterilisation, which was $7.5 \%( \pm 0.8 \%)$, to calculate percentage of anthracene disappearance in M. quercophilus culture. These percentages were 73 and $79 \%$ (standard deviation under 1.5) after 8 and 15-day incubation time respectively. Anthracene oxidation was mainly due to laccase activity alone since neither manganese peroxidase nor lignine peroxidase activities were detected in the culture medium (data not shown). This result corroborates those found in a previous study (Farnet et al., 2004), with another M. quercophilus strain, where no peroxidise activities were detected. Here, the percentage of anthracene disappearance was quite similar after 8- and 15-day incubation time (73 and 79\%). This result may be explained by a peak of laccase production: laccase activity was indeed measured throughout the culture and showed that he highest activity $\left(8 \mathrm{U} \mathrm{L}^{-1}\right)$ was observed after 7 days of culture. Then, laccase activity progressively decreased

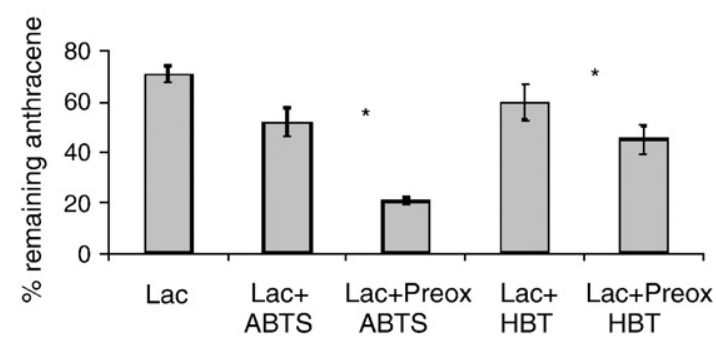

Fig. 3. Anthracene oxidation with the constitutive laccase of Marasmius quercophilus, with or without mediators (ABTS and HBT) and with or without a preoxidation of ABTS and HBT (PREOX_ABTS, PREOX_HBT). Results are expressed as percentages of remaining anthracene detected with HPLC (average of three experiments). Stars indicate significant differences $(P<0.05)$ between means of percentage of remaining anthracene with or without pre-oxidation of the mediator used. 
and after 15 days of culture it was $3 \mathrm{U} \mathrm{L}^{-1}$. The weak solubility of anthracene in water did not affect laccase oxidation of this molecule by decreasing its bioavailability. While acetone was added to the reaction mixture with the purified laccase to favour solubilisation, no solvent was used in fungal cultures to prevent toxic effects of the solvent on the mycelial growth.

The present study highlights the importance of fungal extracellular oxidative enzyme in pollutant degradation. Steffen et al. (2007) have shown the role of various species of litter decomposing fungi in high molecular mass PAH transformation in soil. This transformation was indeed enhanced by fungi (such as Stropharia spp.) while autochthonous microorganisms had a weaker transformation potential (average transformation percentage of 80 and 20 respectively). Furthermore, our study gives evidence of infraspecies laccase diversity which was assessed using isoelectric focusing. This result corroborates those found in previous studies on laccase gene diversity in a forest soil (Luis et al., 2004) and thus, further studies should concentrate in correlating both protein and gene functional diversity. Moreover, it would be valuable to perform in situ extraction of laccases from litter in order to identify, after protein sequencing, the fungi which actually produced laccases in forest litter.

Moreover, this in vitro study has shown that measuring oxidative activities can lead to misinterpretations depending on the conditions used since $\mathrm{PAH}$ are responsible for abiotic reactions towards laccase substrate. In a previous study (Farnet et al., 2008), we have shown that abiotic oxidation or reduction may occur on the laccase substrate with different metal ions. Thus, in complex environments such as soils or litters where many molecules can interact with the enzyme substrate, results on laccase activities and other phenoloxidases should be considered with caution. Further studies should concentrate in determining a laccase substrate which oxidation leads to a stable product to limit at least abiotic reduction.

\section{Acknowledgments}

We would like to thank Mrs Marjorie Sweetko for her very helpful assistance in English language for all these years. This work was financially supported by AQUAPROX (France).

\section{References}

Baiocco, P., Barreca, A.M., Fabbrini, M., Galli, C., Gentili, P., 2003. Promoting laccase activity towards non-phenolic substrates: a mechanistic investigation with some laccase-mediator systems. Org. Biomol. Chem. 1, 191.

Baldrian, P., 2003. Interactions of heavy metals with white-rot fungi. Enzyme Microb. Technol. 32, 78-91.

Bernal-Martinez, A., Carrère, H., Patureau, D., Delgenès, J.P., 2007. Ozone pre-treatment as improver of PAH removal during anaerobic digestion of urban sludge. Chemosphere 68, 1013-1019.

Bezalel, L., Hadar, Y., Fu, P., Freeman, J.P., Cerniglia, C.E., 1996. Initial oxidation products in the metabolism of pyrene, anthracene, fluorene and dibenzothiophene by the white-rot fungus Pleurotus ostreatus. Appl. Environ. Microb. 62, 2554-2559.

Bourbonnais, R., Leech, D., Paice, M.G., 1998. Electrochemical analysis of the interactions of laccase mediators with lignin. BBA-Gen. Subjects 1379, 381-390.

Calcaterra, A., Galli, C., Gentili, P., 2008. Phenolic compounds as likely natural mediators of laccase: a mechanistic assessment. J. Mol. Catal., B Enzym. 51,118-120.

Cho, J., Park, S.J., Lim, J.S., Rhee, Y.H., Shin, K.S., 2002. Oxidation of polycyclic aromatic hydrocarbons by laccase of Coriolus hirsutus. Biotechnol. Lett. 24, 1337-1340.

Collins, P.J., Kotterman, M.J.J., Field, J.A., Dobson, A.D.W., 1996. Oxidation of anthracene and benzo[a]pyrene by laccases from Trametes versicolor. Appl. Environ. Microbiol. 62, 4563-4567.

Dodor, D.E., Hwang, M., Ekunwe, S.I.N., 2004. Oxidation of anthracene and benzo[a] pyrene by immobilized laccase from Trametes versicolor. Enzyme Microb. Technol. 35, 210-217.

Eom, I.C., Rast, C., Veber, A.M., Vasseur, P., 2007. Ecotoxicity of a polycyclic aromatic hydrocarbon (PAH)-contaminated soil. Ecotoxicol. Environ. Saf. 67, 190-205.
Farnet, A.M., Criquet, S., Tagger, S., Gil, G., Le Petit, J., 2000. Purification, partial characterization and reactivity with aromatic compounds of two laccases from Marasmius quercophilus strain 17. Can. J. Microbiol. 46, 189-194.

Farnet, A.M., Criquet, S., Cigna, M., Gil, G., Ferre, E., 2004. Purification of a laccase from Marasmius quercophilus induced with ferulic acid reactivity towards natural and xenobiotic aromatic compounds. Enzyme Microb. Technol. 34, 549-554.

Farnet, A.M., Gil, G., Ferre, E., 2008. Effects of pollutants on laccase activities of Marasmius quercophilus, a white-rot fungus isolated from a Mediterranean schlerophyllous litter. Chemosphere 70, 895-900.

Farnet, A.M., Tagger, S., Le Petit, J., 1999. Effects of copper and aromatic inducers on the laccases of the white-rot fungus Marasmius quercophilus. C.R.A.S., Life Sci. 322, 499-503.

Field, J.A., Vledder, R.H., van Zelst, J.G., Rulkens, W.H., 1996. The tolerance of lignin peroxidase and manganese-dependent peroxidase to miscible solvents and the in vitro oxidation of anthracene in solvent: water mixtures. Enzyme Microb. Technol. $18,300-308$.

Hamdi, H., Manusadžianas, L., Aoyama, I., Jedidi, N., 2006. Effects of anthracene, pyrene and benzo[a]pyrene spiking and sewage sludge compost amendment on soil ecotoxicity during a bioremediation process. Chemosphere 65, 1153-1162.

Hammel, K.E., Kalyanaraman, B., Kirk, T.K., 1986. Oxidation of polycyclic aromatic hydrocarbons and dibenzo[p]-dioxins by Phanerochaete chrysosporium ligninase. J. Biol. Chem. 261, 16948-16952.

Hu, X., Zhang, Y., Zhao, X., Hwang, H.-M., 2007. Biodegradation of benzo[a]pyrene with immobilized laccase: genotoxicity of the products in HaCaT and A3 Cell. Environ. Mol. Mutagen. 48, 106-113.

Laemmli, U.K., 1970. Cleavage of structural proteins during the assembly of the head of bacteriophage T4. Nature 227, 680-685.

Lau, K.L., Tsang, Y.Y., Chiu, S.W., 2003. Use of spent mushroom compost to bioremediate PAH-contaminated samples. Chemosphere 52, 1539-1546.

Luis, P., Walther, G., Kellner, H., Martin, F., Buscot, F., 2004. Diversity of laccase genes from basidiomycete in a forest soil. Soil Biol. Biochem. 36, 1025-1036.

Michniewics, A., Ullrich, R., Ledakowicz, S., Hofrichter, M., 2006. The white-rot fungus Cerrena unicolor strain 137 produces two laccase isoforms with different physicochemical and catalytic properties. Appl. Microbiol. Biotechnol. 69, 682-688.

Mill, T., Mabey, W.R., Lan, B.Y., Baraze, A., 1981. Photolysis of polycyclic hydrocarbons in water. Chemosphere 10, 1281-1284.

Miller, J.S., Olejnik, D., 2000. Photolysis of polycyclic aromatic hydrocarbons in water. Water Res. 35, 233-243.

Muhit, M., Razzaque, R., Grathwohl, P., 2008. Predicting organic carbon-water partitioning of hydrophobic organic chemicals in soils and sediments based on water solubility. Water Res. 42, 3775-3780.

Palmieri, G., Giardina, P., Bianco, C., Fontanella, B., Sannia, G., 2000. Copper induction of laccase isoenzymes in the ligninolytic fungus Pleurotus ostreatus. Appl. Microbiol. Biotechnol. 39, 32-636.

Palmieri, G., Cennamo, G., Faraco, V., Amoresano, A., Sannia, G., Giardina, P., 2003. A typical laccase isoenzyme from copper-supplemented Pleurotus ostreatus cultures. Enzyme Microb. Technol. 33, 220-230.

Pickard, M.A., Roman, R., Tinoco, R., Vazquez-Duhalt, R., 1999. Polycyclic Aromatic Hydrocarbon metabolism by white-rot fungi and oxidation by Coriolopsis gallica UAMH 8260 Laccase. Appl. Environ. Microbiol. 65, 3805-3809.

Potin, O., Veignie, E., Rafin, C., 2004. Biodegradation of polycyclic aromatic hydrocarbons (PAH) by Cladosporium sphaerospermum isolated from an aged $\mathrm{PAH}$ contaminated soil. FEMS Microbiol. Ecol. 51, 71-78.

Pozdnyakova, N.N., Rodakiewics-Nowak, J., Turkovskaya, O.V., Haber, J., 2006. Oxidative degradation of polyaromatic hydrocarbons catalyzed by blue laccase from Pleurotus ostreatus D1 in the presence of synthetic mediators. J. Mol. Catal., B Enzym. 41, 8-15.

Racon, L., Sadaka, N., Gil, G., Le Petit, J., Matheron, R., Poinsot-Balaguer, N., Sigoillot, J.C., Woltz, P., 1988. Histological and chemical changes in tannic compounds of evergreen oak leaf litter. Can. J. Bot. 66, 663-667.

Rodríguez, E., Nuero, O., Guillén, F., Martínez, A.T., Martínez, M.J., 2004. Degradation of phenolic and non-phenolic aromatic pollutants by four Pleurotus species: the role of laccase and versatile peroxidase.Soil Biol. Biochem. 36, 909-916.

Roseen, P.A., Gubanski, S.M., Gedde, U.W., 1995. External partial discharge resistance of polyethylene and cross linked polyethylene containing voltage stabilizers. Electrical Insulation and Dielectric Phenomena, 1995. Annual Report, pp. 247-249. 22-25 Oct.

Stajic, M., Persky, L., Friesem, D., Hadar, Y., Wasser, P.S., Eviatar-Nevo, E., Vukojevic, J., 2006. Effect of different carbon and nitrogen sources on laccase and peroxidise production by selected Pleurotus species. Enzyme Microb. Technol. 38, 65-73.

Steffen, K.T., Shuvbert, S., Tuomela, M., Hatakka, A., Hofricht, M., 2007. Enhancement of bioconversion of high molecular mass polycyclic aromatic hydrocarbon in contaminated non-sterile soils by litter-decomposing fungi. Biodegradation 18, 359-369.

Valentín, L., Feijoo, G., Moreira, M.T., Lema, J.M., 2006. Biodegradation of polycyclic aromatic hydrocarbons in forest and salt marsh soils by white-rot fungi. Int. Biodeterior. Biodegrad. 58, 15-21.

Zhang, J., Liu, X., Xu, Z., Chen, H., Yang, Y., 2008. Degradation of chlorophenols catalyzed by laccase. Int. Biodeterior. Biodegrad. 61, 351-356. 